\title{
Valence-Only Correlation in LiH and $\mathrm{BeH}^{+*}$
}

\author{
W. A. Sanders** \\ The Catholic University of America, Washington, D.C. 20017
}

M. Krauss

Institute for Basic Standards, National Bureau of Standards, Washington, D.C. 20234

(October 9, 1967)

\begin{abstract}
The pseudonatural orbital procedure has been applied to the calculation of the potential energy curve of $\mathrm{LiH}$ and the dissociation energy of $\mathrm{BeH}^{+}$. Only the two-electron bonding pair is correlated and estimates of $\sigma$ and $\pi$ type correlation are obtained. The results for $\mathrm{LiH}$ are in good agreement with the most accurate previously published calculations. Comparison with experimental results for $\mathrm{LiH}$ indicates that the calculated dissociation energies are accurate to about 0.15 to $0.2 \mathrm{eV}$.
\end{abstract}

Key Words: $\mathrm{BeH}^{+}$, correlation energy, dissociation energy, LiH, molecular orbital, potential energy curve.

\section{Introduction}

The calculation of accurate potential energy curves of small molecules cannot be achieved within the framework of the Hartree-Fock one-electron model. Consideration of the correlation is necessary to insure both the correct asymptotic dependence and the depth and shape of the curve near the equilibrium separation. In order to simplify the problem correlation considerations should be limited to only those electrons intimately involved in the binding process, i.e., the valence electrons. This permits the utilization of localization techniques which limit the number of relevant electrons that must be correlated. Prior to the introduction of localization techniques it is necessary to show that, as expected, only the valence electrons need be considered. Evidence for the validity of this concept has been accumulated by extended Hartree-Fock (H. F.) calculations $[1]^{1}$ on the molecules $\mathrm{H}_{2}$ and $\mathrm{Li}_{2}$, for which the bonding valence electrons are a simple pair. Additional work has been done on systems for which intershell pairs are significant [2]. However, such complicated systems fall beyond the range of the present investigation, which is to add to the evidence for the accuracy of potential curves calculated by correlating only the two-electron valence shell.

* Acknowledgement is made to the donors of the Petroleum Research Fund. administered by the American Chemical Society. for partial support of this research.

The Catholic University of America. 620 Michigan Ave.. N.E.. Washington. D.C. 20017. ' Figures in brackets indicate the literature references at the end of this paper.
The simplest case beyond $\mathrm{H}_{2}$ is the isoelectronic $\mathrm{LiH}$ sequence. Numerous calculations [3] of this molecule have appeared over the years but the relative simplicity of the model has enticed all the investigators to do as complete a calculation as possible within their model. Those studies which consider correlation therefore apply a correlated trial function to both the Li $K$ shell and the bonding shell. Although the probability of success for a valence-shell-only treatment is predictably high, we feel that such a calculation is required as a basis for future work in this area.

The calculation in this paper is equivalent to the extended H. F. calculation with a frozen $l \sigma(K$ shell $)$ H. F. molecular orbital. The actual procedure follows the pseudonatural orbital (PNO) procedure previously applied to the three-electron system [2]. In effect the virtual $H$. F. orbitals are transformed into approximate natural orbitals [4] which span the same region of space as the occupied H. F. valence orbitals. Such orbitals provide for rapid convergence in the superposition of configurations (SOC) calculation. A number of points of the $\mathrm{LiH}$ curve were calculated in this way. The results are compared in particular to the natural orbital based calculation of Bender and Davidson [3f] which is the most accurate calculation for $\mathrm{LiH}$. We can anticipate our conclusion by noting that the outer-shell correlation energies for the two calculations are quite comparable.

The isoelectronic system $\mathrm{BeH}^{+}$is also considered only at the equilibrium internuclear separation. Since the electronic distributions in the H. F. solution for $\mathrm{LiH}$ and $\mathrm{BeH}^{+}$are quite different, the correlation 
results shed light on different bonding cases ranging from the ionic or strongly polarized through the covalent. For the $\mathrm{LiH}$ and $\mathrm{BeH}^{+}$molecules the techniques utilized have proved convenient and quantitative.

\section{Pseudonatural Orbitals and Basis Functions} form

All calculations are based on trial functions of the

$$
\psi(1,2,3,4)=A \psi_{1}(1) \psi_{1}(2) \psi(3,4)
$$

where $\psi_{1}$ is an accurate approximation to the $1 \sigma \mathrm{H} . \mathrm{F}$. molecular orbital, $\varphi$ is a two-electron pair function including a singlet spin function, and $A$ is the antisymmetrizer. The pair function is held strongly orthogonal to the $\psi_{1}$ orbital. The overall symmetry is ${ }^{1} \Sigma+$ and the wave function would agree with the result of an extended H. F. calculation if the $\psi_{1}$ orbital were permitted to relax in the field of the pair function.

The $\varphi(3,4)$ is determined here in two steps. Using the $\sigma$ virtual H. F. solutions, a superposition of all configurations formed by all single and double excitations from the valence shell molecular orbital is diagonalized. Similarly, an arbitrarily orthonormalized set of $\pi$ orbitals is diagonalized. The first-order density matrices for both the $\sigma$ and $\pi$ substituted wave functions are diagonalized by determining the equivalent transformation which diagonalized the coefficient matrix for the SOC expansion. The approximate or pseudonatural orbitals are now available for a complete SOC involving both $\sigma$ and $\pi$ excitations. Only diagonal excitations are now considered and the final SOC requires a limited number of interaction integrals in the new basis, thereby simplifying the calculation.

The use of pseudonatural orbitals is most convenient when intershell pairs are significant. Extended H. F. procedures would actually be better in the present case, since only one pair is being correlated. However, the results are essentially equivalent and are obtained with comparable effort.

The basis set is built around the best atom Gaussiantype function (GTF) H. F. solutions of Huzinaga [5]. As is widely known [6], the natural orbitals are localized in the same region of space as the respective $\mathrm{H}$. F. orbitals. Except for polarization orbitals of new symmetry type the H. F. basis should suffice. Polarization orbitals were added by scaling GTF fits to $p \sigma$ and $p \pi$ functions and varying the scale factor to determine the best energy. The variations were by no means exhaustive and improvements are possible. The variations were done only for $\mathrm{LiH}$ at 3.0 a.u. and the same basis was used for the entire curve (or appropriately scaled for the $\mathrm{BeH}^{+}$case).

Small exponent $s$ and $p$ GTF are centered on the $\mathrm{Li}$ atom. They are intended to account for the polarization of the charge distribution toward the $\mathrm{H}$ atom. Whether the $\mathrm{LiH}$ charge density can be represented by an ionic model is somewhat debatable [7] with the present evidence leaning toward the polarization of a diffuse $\mathrm{Li} 2 s$ function. The large set on the Li actually contributes to the wavefunction near $\mathrm{H}$ with the heavy weight to the small exponent functions.

The accuracy of the basis is best judged in this light by comparison with the results using significantly different bases. The $\mathrm{BeH}^{+}$charge density has even less resemblance to an ionic model and the basis should be adequate.

The basis sets are defined in table 1 and the PNO coefficients are given in table 2 . The first two orbitals are exactly the undisturbed $1 s_{\mathrm{Li}} \mathrm{H}$. F. molecular orbital and the bonding PNO which is very similar to the second occupied H.F. molecular orbital. This orbital has been referred to as a $1 s_{\mathrm{H}^{-}}$orbital because of the ionicity of the $\mathrm{LiH}$ bond [8]. However, the $2 s_{\mathrm{Li}}$ and $2 p_{\mathrm{Li}}$ densities are far from insignificant. From our results and those of Bender and Davidson on the partitioning of the correlation into $\sigma$ and $\pi$ type terms, the identification of this orbital as a $1 s_{\mathrm{H}^{-}}$is obviously oversimplified. The basis set that was chosen to represent both the $\mathrm{H}$. F. and correlation may, however, underestimate the need for polarization of the $\mathrm{H}$ centered density.

TABle 1. Parameters for basis orbitals, $\mathrm{f}(\mathrm{x}, \mathrm{y}, \mathrm{z}) \exp \left(-\mathrm{al}^{-2}\right)$

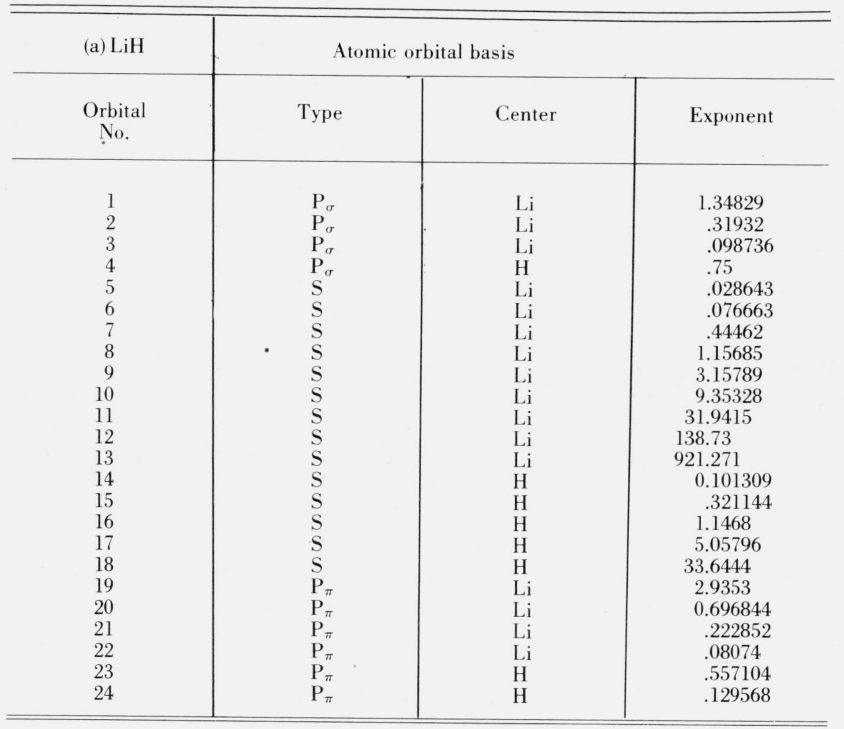

(b) $\mathrm{BeH}^{+}$ Atomic orbital basis

\begin{tabular}{r|r|r|r}
1 & & & \\
2 & $\mathrm{P}_{\sigma}$ & $\mathrm{Be}$ & 1.483 \\
3 & $\mathrm{P}_{\sigma}$ & $\mathrm{Be}$ & .351 \\
4 & $\mathrm{P}_{\sigma}$ & $\mathrm{Be}$ & .109 \\
5 & $\mathrm{P}_{\sigma}$ & $\mathrm{H}$ & .75 \\
6 & $\mathrm{~S}$ & $\mathrm{Be}$ & .0583 \\
7 & $\mathrm{~S}$ & $\mathrm{Be}$ & .1806 \\
8 & $\mathrm{~S}$ & $\mathrm{Be}$ & .8589 \\
9 & $\mathrm{~S}$ & $\mathrm{Be}$ & 2.1847 \\
10 & $\mathrm{~S}$ & $\mathrm{Be}$ & 5.9326 \\
11 & $\mathrm{~S}$ & $\mathrm{Be}$ & 17.6239 \\
12 & $\mathrm{~S}$ & $\mathrm{Be}$ & 60.3255 \\
13 & $\mathrm{~S}$ & $\mathrm{Be}$ & 262.139 \\
14 & $\mathrm{~S}$ & $\mathrm{Be}$ & 1741.38 \\
15 & $\mathrm{~S}$ & $\mathrm{H}$ & 0.1013 \\
16 & $\mathrm{~S}$ & $\mathrm{H}$ & .3211 \\
17 & $\mathrm{~S}$ & $\mathrm{H}$ & 1.1468 \\
18 & $\mathrm{~S}_{1}$ & $\mathrm{H}$ & 5.0579 \\
19 & $\mathrm{~S}_{20}$ & $\mathrm{H}$ & 33.6444 \\
21 & $\mathrm{P}_{\pi}$ & $\mathrm{Be}$ & 3.22883 \\
22 & $\mathrm{P}_{\pi}$ & $\mathrm{Be}$ & .245628 \\
23 & $\mathrm{P}_{\pi}$ & $\mathrm{Be}$ & .088814 \\
24 & $\mathrm{P}_{\pi}$ & $\mathrm{Be}$ & .557104 \\
& $\mathrm{P}_{\pi}$ & $\mathrm{H}$ & .129568 \\
\hline
\end{tabular}


TABLE 2. Expansion coefficients for the Hartree-Fock and pseudo-

1 a.u. (length) $=0.529172 \mathrm{~A}$

(a) PNO Coefficients. $\mathrm{LiH} R=2.0$

\begin{tabular}{|c|c|c|c|c|c|c|}
\hline $\begin{array}{c}\text { Orbital } \\
\text { No. }\end{array}$ & $\mathrm{l} \sigma \mathrm{H} . \mathrm{F}$. & $2 \sigma \mathrm{H} . \mathrm{F}$. & $2 \sigma$ & $3 \sigma$ & $4 \sigma$ & $5 \sigma$ \\
\hline $\begin{array}{r}1 \\
2 \\
3 \\
4 \\
5 \\
6 \\
7 \\
8 \\
9 \\
10 \\
11 \\
12 \\
13 \\
14 \\
15 \\
16 \\
17 \\
18\end{array}$ & $\begin{array}{r}0.004407 \\
.007878 \\
-.002017 \\
.004964 \\
-.000002 \\
.002588 \\
.167498 \\
.424381 \\
.341843 \\
.160068 \\
.049580 \\
.010380 \\
.001360 \\
-.001627 \\
.010437 \\
.009115 \\
.002351 \\
.000373\end{array}$ & $\begin{array}{r}0.033912 \\
.091818 \\
.117561 \\
-.021768 \\
-.073225 \\
-.172702 \\
.097015 \\
.118228 \\
.066803 \\
.029541 \\
.008663 \\
.001814 \\
.000235 \\
-.317569 \\
-.360808 \\
-.164161 \\
-.038021 \\
-.005348\end{array}$ & $\begin{array}{r}0.034077 \\
.088182 \\
.114025 \\
-.021483 \\
-.099539 \\
-.174916 \\
.095932 \\
.118447 \\
.067831 \\
.029685 \\
.008762 \\
.001826 \\
.000237 \\
-.310838 \\
-.346714 \\
-.173403 \\
-.038413 \\
-.005422\end{array}$ & $\begin{array}{r}0.008329 \\
-.068465 \\
-.231170 \\
-.072883 \\
.252490 \\
. .317555 \\
-.069496 \\
.015033 \\
.003539 \\
.003243 \\
.000617 \\
.000175 \\
.000018 \\
.625276 \\
-.794154 \\
-.447228 \\
-.054958 \\
-.008110\end{array}$ & $\begin{array}{r}-0.032827 \\
-.477243 \\
-.554928 \\
.484559 \\
-.225179 \\
1.704137 \\
.568379 \\
-.533391 \\
.002012 \\
-.069599 \\
-.007569 \\
-.003246 \\
-.000279 \\
-1.598575 \\
-.378740 \\
.012201 \\
-.010818 \\
.000229\end{array}$ & $\begin{array}{r}-0.017292 \\
-.012876 \\
.367876 \\
.234911 \\
-.323240 \\
-.693758 \\
-.050188 \\
-.122566 \\
-.007132 \\
-.017212 \\
-.002056 \\
-.000788 \\
-.000069 \\
.257708 \\
1.736012 \\
-1.266605 \\
-0.165061 \\
-.010251\end{array}$ \\
\hline 18 & $1 \pi$ & $2 \pi$ & & & & \\
\hline $\begin{array}{l}19 \\
20 \\
21 \\
22 \\
23 \\
24\end{array}$ & $\begin{array}{r}0.006254 \\
.010794 \\
.103205 \\
-.049588 \\
.499923 \\
.591811\end{array}$ & $\begin{array}{r}0.017242 \\
.047861 \\
.130557 \\
-.026074 \\
-1.089518 \\
.934826\end{array}$ & & & & \\
\hline
\end{tabular}

(b) PNO Coefficients, LiH $R=2.6$

\begin{tabular}{|c|c|c|c|c|c|c|}
\hline 1 & 0.006374 & 0.022623 & 0.022271 & 0.006199 & -0.028556 & -0.014421 \\
\hline 2 & .005588 & .078845 & .077196 & .059848 & -.398499 & -.111385 \\
\hline 3 & -.001458 & .107365 & .102291 & .254043 & -.428187 & .458737 \\
\hline 4 & .001660 & -.018462 & -.017715 & .052101 & .447592 & .198343 \\
\hline 5 & -.000365 & -.076565 & -.100904 & -.249082 & -.231610 & -.327268 \\
\hline 6 & .002908 & -.196271 & -.203380 & -.484031 & 1.190100 & -.833528 \\
\hline 7 & 171372 & .053235 & .051759 & .101569 & .385691 & .224806 \\
\hline 8 & .423021 & .097091 & .099854 & .048487 & -.376673 & -.134147 \\
\hline 9 & .343919 & .048569 & .049199 & .027676 & -.011197 & .009049 \\
\hline 10 & .160316 & .022722 & .023160 & .011783 & -.050662 & -.015508 \\
\hline 11 & .049759 & .006484 & .006589 & .003410 & -.006656 & -.000927 \\
\hline 12 & .010401 & .001382 & .001406 & .000710 & -.002475 & -.000667 \\
\hline 13 & .001364 & .000177 & .000180 & .000091 & -.000229 & -.000045 \\
\hline 14 & -.000849 & -.342670 & -.328782 & -.458568 & -1.339842 & .595913 \\
\hline 15 & .004437 & -.334963 & -.325985 & .829587 & -.000825 & 1.280689 \\
\hline 16 & .002763 & -.147001 & -.155785 & .383794 & .033370 & -1.174829 \\
\hline 17 & .000597 & -.035569 & -.035967 & .053933 & -.007512 & -0.127366 \\
\hline \multirow[t]{2}{*}{18} & .000109 & -.004919 & -.005002 & .007591 & .000534 & -.010907 \\
\hline & $1 \pi$ & $2 \pi$ & & & & \\
\hline 19 & 0.005296 & -0.009818 & & & & \\
\hline 20 & .005507 & -.002790 & & & & \\
\hline 21 & .154002 & -.187711 & & & & \\
\hline 22 & -.011169 & -.115084 & & & & \\
\hline 23 & .449336 & 1.057181 & & & & \\
\hline 24 & .599113 & -0.739935 & & & & \\
\hline
\end{tabular}

1 a.u. (length) $=0.529172 \AA$

(c) PNO Coefficients, $\mathrm{LiH} R=3.0$

\begin{tabular}{|c|c|c|c|c|c|c|}
\hline $\begin{array}{c}\text { Orbital } \\
\text { No. }\end{array}$ & $l \sigma \mathrm{H} . \mathrm{F}$. & $2 \sigma$ H. F. & $2 \sigma$ & $3 \sigma$ & $4 \sigma$ & $5 \sigma$ \\
\hline $\begin{array}{r}1 \\
2 \\
3 \\
4 \\
5 \\
6 \\
7 \\
8 \\
9 \\
9 \\
10 \\
11 \\
12 \\
13 \\
14 \\
15 \\
16 \\
17 \\
18\end{array}$ & $\begin{array}{r}0.006264 \\
.005319 \\
-.001506 \\
.000962 \\
-.000437 \\
.002998 \\
.170797 \\
.423435 \\
.344369 \\
.160459 \\
.049808 \\
.010409 \\
.001366 \\
-.000747 \\
.002781 \\
.001290 \\
.000281 \\
.000050\end{array}$ & $\begin{array}{r}0.016858 \\
.069495 \\
.107464 \\
-.016321 \\
-.085497 \\
-.207120 \\
.042737 \\
.086114 \\
.039994 \\
.019650 \\
.005456 \\
.001184 \\
.000150 \\
-.364782 \\
-.318529 \\
-.140493 \\
-.034264 \\
-.004698\end{array}$ & $\begin{array}{r}0.016266 \\
.068472 \\
.102147 \\
-.014973 \\
-.109990 \\
-.220749 \\
.042031 \\
.089866 \\
.040816 \\
.020300 \\
.005598 \\
.001220 \\
.000154 \\
-.342419 \\
-.313586 \\
-.149304 \\
-.034721 \\
-.004787\end{array}$ & $\begin{array}{r}0.011714 \\
.067528 \\
.276211 \\
.018269 \\
-.249975 \\
-.576710 \\
.092888 \\
.083038 \\
.040843 \\
.018641 \\
.005257 \\
.001118 \\
.000143 \\
-.303882 \\
.814402 \\
.338235 \\
.052848 \\
.007121\end{array}$ & $\begin{array}{r}-0.007152 \\
-.380256 \\
-.378214 \\
.443654 \\
-.280662 \\
.996402 \\
.323136 \\
-.326297 \\
.003043 \\
-.041905 \\
-.004722 \\
-.001997 \\
-.000174 \\
-1.304840 \\
0.245100 \\
.060241 \\
.003527 \\
.001194\end{array}$ & $\begin{array}{r}-0.000853 \\
.174121 \\
-.434746 \\
-.231176 \\
.370475 \\
.728993 \\
-.289679 \\
.143809 \\
-.030252 \\
.012447 \\
-.000831 \\
.000431 \\
.000005 \\
-.654657 \\
-1.045312 \\
1.112123 \\
0.105760 \\
.011175\end{array}$ \\
\hline & $1 \pi$ & $2 \pi$ & & & & \\
\hline $\begin{array}{l}19 \\
20 \\
21 \\
22 \\
23 \\
24\end{array}$ & $\begin{array}{r}0.005663 \\
-.005169 \\
.161447 \\
.022901 \\
.413339 \\
.622294\end{array}$ & $\begin{array}{r}-0.007912 \\
.005612 \\
-.209146 \\
-.193739 \\
1.031171 \\
-0.630077\end{array}$ & & & & \\
\hline
\end{tabular}

(d) PNO Coefficients, $\mathrm{LiH} R=3.4$

\begin{tabular}{|c|c|c|c|c|c|c|}
\hline 1 & 0.005777 & 0.012993 & 0.012383 & -0.012011 & 0.015253 & 0.018591 \\
\hline 2 & .005095 & .058102 & .057180 & -.067785 & -.353559 & $\begin{array}{r}-.198672 \\
-.1967\end{array}$ \\
\hline 3 & -.001441 & -110553 & . 104984 & -.285139 & -.378649 & .347227 \\
\hline 4 & .000646 & -.014262 & -.012337 & .004920 & .447394 & .291657 \\
\hline 5 & -.000436 & -.097410 & -.123429 & .255716 & -.340258 & -.422054 \\
\hline 6 & .002847 & -.213738 & -.235591 & .615901 & .903941 & -.562947 \\
\hline 7 & .170049 & .040677 & .041490 & -.088486 & .236146 & .272905 \\
\hline 8 & .423967 & .075020 & . 079468 & -.100022 & -.268000 & -.129519 \\
\hline 9 & .344544 & .034459 & .035914 & -.046361 & .014584 & .047560 \\
\hline 10 & .160571 & .017156 & .018024 & -.022163 & -.034262 & -.008843 \\
\hline 11 & .049833 & .004721 & .004935 & -.006135 & -.002916 & .002546 \\
\hline 12 & .010415 & .001030 & .001080 & -.001324 & -.001572 & -.000172 \\
\hline 13 & .001366 & .000130 & .000136 & -.000168 & -.000123 & .000036 \\
\hline 14 & -.000574 & -.388998 & -.356436 & .184030 & -1.297227 & .650819 \\
\hline 15 & .001807 & -.302829 & -.302215 & -.768513 & 0.438748 & .853499 \\
\hline 16 & .000598 & -.136544 & -.145546 & -.296663 & .073463 & -1.058826 \\
\hline 17 & .000151 & -.033149 & -.033677 & -.050383 & .014132 & -0.088454 \\
\hline \multirow[t]{2}{*}{18} & .000023 & -.004542 & -.004638 & -.006625 & .001653 & -.011337 \\
\hline & $1 \pi$ & $2 \pi$ & & & & \\
\hline 19 & 0.005932 & 0.007031 & & & & \\
\hline 20 & -.012050 & -.009586 & & & & \\
\hline 21 & .149304 & .216540 & & & & \\
\hline 22 & .063263 & .263758 & & & & \\
\hline 23 & .382200 & -1.000147 & & & & \\
\hline 24 & 646210 & 0.545015 & & & & \\
\hline
\end{tabular}


TABLE 2. Expansion coefficients for the Hartree-Fock and pseudonatural orbitals-Continued

1 a.u. (length) $=0.529172 \AA$

(e) PNO Coefficients, $\mathrm{LiH} R=4.0$

\begin{tabular}{|c|c|c|c|c|c|c|}
\hline $\begin{array}{c}\text { Orbital } \\
\text { No. }\end{array}$ & $\mathrm{l} \sigma \mathrm{H} . \mathrm{F}$. & $2 \sigma$ H. F. & $2 \sigma$ & $3 \sigma$ & $4 \sigma$ & $5 \sigma$ \\
\hline $\begin{array}{r}1 \\
2 \\
3 \\
4 \\
5 \\
6 \\
7 \\
8 \\
8 \\
9 \\
10 \\
11 \\
12 \\
13 \\
14 \\
15 \\
16 \\
17 \\
18\end{array}$ & $\begin{array}{r}0.004917 \\
.004625 \\
-.001250 \\
.000359 \\
-.000404 \\
.002553 \\
.169221 \\
.424585 \\
.344632 \\
.160685 \\
.049852 \\
.010420 \\
.001367 \\
-.000350 \\
.000985 \\
.000153 \\
.000070 \\
.000008\end{array}$ & $\begin{array}{r}0.009900 \\
.039332 \\
.116407 \\
-.010693 \\
-.119925 \\
-.215459 \\
.042781 \\
.060143 \\
.029858 \\
.014223 \\
.004007 \\
.000861 \\
.000109 \\
-.426049 \\
-.281052 \\
-.134137 \\
-.031798 \\
-.004401\end{array}$ & $\begin{array}{r}0.009271 \\
.038823 \\
.108802 \\
-.007997 \\
-.151321 \\
-.251724 \\
.046063 \\
.066555 \\
.032587 \\
.015584 \\
.004378 \\
.000942 \\
.000120 \\
-.375174 \\
-.288216 \\
-.143327 \\
-.032398 \\
-.004500\end{array}$ & $\begin{array}{r}-0.010910 \\
-.048059 \\
-.276563 \\
.015361 \\
.282965 \\
. .606841 \\
-.096618 \\
-.097040 \\
-.050435 \\
-.022909 \\
-.006559 \\
-.001388 \\
-.000178 \\
-.082667 \\
-.693330 \\
-.248696 \\
-.046355 \\
-.005993\end{array}$ & $\begin{array}{r}0.026950 \\
-.251935 \\
-.471289 \\
.455548 \\
-.429119 \\
.890824 \\
.044140 \\
-.137987 \\
.003492 \\
-.020659 \\
-.002060 \\
-.000976 \\
-.000081 \\
-1.273220 \\
0.596246 \\
.082459 \\
.025582 \\
.001990\end{array}$ & $\begin{array}{r}0.031569 \\
-.134434 \\
.122146 \\
.370528 \\
-.503933 \\
-.275745 \\
.117570 \\
-.028820 \\
.042146 \\
.001788 \\
.003494 \\
.000312 \\
.000075 \\
.631880 \\
.572505 \\
-.966482 \\
-.071228 \\
-.01+196\end{array}$ \\
\hline & $1 \pi$ & $2 \pi$ & & & & \\
\hline $\begin{array}{l}19 \\
20 \\
21 \\
22 \\
23 \\
24\end{array}$ & $\begin{array}{r}0.005310 \\
-.012126 \\
.108804 \\
.123893 \\
.347961 \\
.677194\end{array}$ & $\begin{array}{r}0.006601 \\
-.013210 \\
.203931 \\
.363187 \\
-.950778 \\
.458483\end{array}$ & & & & \\
\hline
\end{tabular}

(f) PNO Coefficients, $\mathrm{LiH} R=6.0$

\begin{tabular}{|c|c|c|c|c|c|c|}
\hline 1 & 0.002703 & -0.006172 & -0.004003 & 0.005354 & 0.025793 & -0.013727 \\
\hline 2 & .002877 & -.004471 & -.006218 & .003205 & -.152044 & .095237 \\
\hline 3 & -.000616 & -.111914 & -.072472 & .121849 & .740686 & -.312469 \\
\hline 4 & -.000011 & .005416 & .001766 & -.005371 & -.553481 & .274310 \\
\hline 5 & -.000319 & .218056 & .289034 & -.418685 & .450527 & -.430933 \\
\hline 6 & .001914 & 179907 & 298790 & -.467353 & -.695251 & .060749 \\
\hline 7 & .168348 & -.039456 & -.058646 & .090123 & .253192 & -.100854 \\
\hline 8 & .425325 & -.041442 & -.060795 & .085498 & -.114445 & .115331 \\
\hline 9 & .344690 & -.023180 & -.033747 & .048901 & .054881 & -.006532 \\
\hline 10 & .160819 & -.010249 & -.014897 & .021093 & -.007640 & .016090 \\
\hline 11 & .049869 & -.003010 & -.004373 & .006273 & .003538 & .001180 \\
\hline 12 & .010426 & -.000629 & -.000913 & .001298 & -.000057 & .000733 \\
\hline 13 & .001367 & -.000081 & -.000118 & .000169 & .000059 & .000054 \\
\hline 14 & -.000004 & .520188 & .361914 & .104801 & .898639 & .897501 \\
\hline 15 & .000089 & .240391 & .292588 & .496591 & -.550843 & -.220667 \\
\hline 16 & .000033 & .136112 & .142144 & .168076 & -.130697 & -.646153 \\
\hline 17 & .000009 & .029438 & .030877 & .037554 & -.029655 & -.052456 \\
\hline \multirow[t]{2}{*}{18} & .000001 & .004280 & .004301 & .004759 & -.002595 & -.009329 \\
\hline & $1 \pi$ & & & & & \\
\hline 19 & 0.000790 & & & & & \\
\hline 20 & .007953 & & & & & \\
\hline 21 & -.010902 & & & & & \\
\hline 22 & .197279 & & & & & \\
\hline 23 & .326293 & & & & & \\
\hline 24 & .739552 & & & & & \\
\hline
\end{tabular}

1 a.u. (length) $=0.529172 \AA$

(g) PNO Coefficients, $\mathrm{LiH} R=8.0$

\begin{tabular}{|c|c|c|c|c|}
\hline $\begin{array}{c}\text { Orbital } \\
\text { No. }\end{array}$ & $\mathrm{l} \sigma \mathrm{H} . \mathrm{F}$. & $2 \sigma$ H. F. & $2 \sigma$ & 3 \\
\hline $\begin{array}{r}1 \\
2 \\
3 \\
4 \\
4 \\
5 \\
6 \\
7 \\
8 \\
9 \\
10 \\
11 \\
12 \\
13 \\
14 \\
15 \\
16 \\
17 \\
18\end{array}$ & $\begin{array}{r}-0.001449 \\
-.001564 \\
.000283 \\
-.000001 \\
-.000307 \\
.001755 \\
.168259 \\
.425437 \\
.344690 \\
.160840 \\
.049870 \\
.010429 \\
.001366 \\
-.000006 \\
.000055 \\
-.000002 \\
.000003 \\
.000000\end{array}$ & $\begin{array}{r}0.004125 \\
-.006912 \\
.084680 \\
-.003467 \\
.306383 \\
.147139 \\
-.036650 \\
-.041815 \\
-.022221 \\
-.010078 \\
-.002912 \\
-.000615 \\
-.000078 \\
.571740 \\
.219115 \\
.140310 \\
.028597 \\
.004308\end{array}$ & $\begin{array}{r}0.000824 \\
.000074 \\
.016580 \\
-.000248 \\
.365248 \\
.355322 \\
-.069391 \\
-.071124 \\
-.039241 \\
-.017341 \\
-.005082 \\
-.001062 \\
-.000137 \\
.296693 \\
.329643 \\
.142635 \\
.031340 \\
.004262\end{array}$ & $\begin{array}{r}-0.000916 \\
.000871 \\
-.019912 \\
.000412 \\
-.415246 \\
-.414143 \\
.080760 \\
.081196 \\
.045218 \\
.019845 \\
.005839 \\
.001217 \\
.000158 \\
.227271 \\
.398485 \\
.150219 \\
.033858 \\
.004430\end{array}$ \\
\hline & $1 \pi$ & & & \\
\hline $\begin{array}{l}19 \\
20 \\
21 \\
22 \\
23 \\
24\end{array}$ & $\begin{array}{r}-0.000368 \\
.009481 \\
-.028502 \\
.134216 \\
.349310 \\
.755300\end{array}$ & & & \\
\hline
\end{tabular}

(h) PNO Coefficients, $\mathrm{BeH}^{+} R=2.48$

\begin{tabular}{c|r|r|r|r|r|r}
\hline $\begin{array}{c}\text { Orbital } \\
\text { No. }\end{array}$ & $1 \sigma \mathrm{H} . \mathrm{F}$. & $2 \sigma \mathrm{H} . \mathrm{F}$. & $2 \sigma$ & $3 \sigma$ & \multicolumn{1}{c}{$4 \sigma$} & \multicolumn{1}{c}{$5 \sigma$} \\
\hline & & & & & & \\
1 & 0.007213 & 0.047173 & 0.045504 & -0.015329 & -0.113542 & 0.061338 \\
2 & -.000225 & .147334 & .137219 & -.176527 & -.470720 & .249390 \\
3 & .000239 & .085880 & .074154 & -.131239 & .149033 & -.043572 \\
4 & .000591 & -.033569 & -.031121 & -.093298 & .466382 & .058923 \\
5 & .000557 & .103897 & .107463 & -.182799 & .339832 & -.338659 \\
6 & -.002563 & .434705 & .447353 & -.912291 & -.332627 & -.369361 \\
7 & -.161356 & -.044723 & -.042953 & .070612 & -.384425 & .136442 \\
8 & -.425490 & -.120599 & -.124988 & .148209 & .319595 & -.044554 \\
9 & -.351194 & -.056745 & -.057854 & .065945 & -.009526 & .018136 \\
10 & -.158475 & -.025758 & -.026487 & .030270 & .037430 & .000387 \\
11 & -.047991 & -.007002 & -.007162 & .008003 & .003327 & .001269 \\
12 & -.009950 & -.001494 & -.001533 & .001724 & .001667 & .000106 \\
13 & -.001302 & -.000189 & -.000194 & .000216 & .000133 & .000024 \\
14 & -.000128 & .103976 & .102979 & -.068896 & .506348 & .175235 \\
15 & -.000943 & .341322 & .335900 & .760629 & -.008797 & 1.146932 \\
16 & -.000627 & .142022 & .150177 & .385022 & -.105262 & -1.170663 \\
17 & -.000144 & .034818 & .035313 & .049967 & .016663 & -0.117906 \\
18 & -.000023 & .004820 & .004899 & .007649 & -.002056 & -.011233 \\
\hline & & & & & & \\
& $1 \pi$ & $2 \pi$ & & & & \\
\hline 19 & 0.015553 & 0.011738 & & & & \\
20 & .115097 & .138439 & & & & \\
21 & .500244 & .582119 & & & & \\
22 & -.067143 & -.002065 & & & & \\
23 & .439962 & -.958014 & & & & \\
\hline & .304166 & .204944 & & & & \\
\hline
\end{tabular}


The final wavefunctions are obtained with a trial function based on the PNO. The square of the coefficients are listed in table 3. Since only one pair of electrons is excited the $\mathrm{C}^{2}$ are equivalent to the occupation number for the natural spin orbital. Only configurations with $\mathrm{C}^{2}>0.0001$ are included.

\section{Analysis of the Correlation}

The initially occupied $1 \sigma$ and $2 \sigma \mathrm{H}$. F. orbitals are listed in table 2. Comparison of the $2 \sigma \mathrm{H}$. F. orbitals with the $2 \sigma \mathrm{NO}$ shows no appreciable difference until $\mathrm{R}$ exceeds 6 a.u. The first NO and the Hartree-Fock orbital are very similar at the equilibrium configuration [9].

The asymptotic behavior of the $2 \sigma$ and $3 \sigma$ PNO results in the correct atom products, the $\mathrm{H}$. F. ground states of $\mathrm{H}$ and $\mathrm{Li}$. No other $\sigma \mathrm{PNO}$ contributes to the correlation as $\mathrm{R}$ approaches infinity. Of the $\pi$ orbitals only the $1 \pi$ PNO still contributes significantly at 8 a.u.

The $3 \sigma$ PNO in the neighborhood of the equilibrium separation acts mostly as an in-out type of correlation on the $\mathrm{H}^{-}$bonding distribution. Bender and Davidson note that their $3 \sigma$ orbital resembles a $2 s$ hydrogen atom orbital orthogonalized to the $1 s \mathrm{Li}$ orbital. In our case the Li portion of the $3 \sigma$ orbital resembles that part of the $2 \sigma$ which serves as a hybridized $2 s \mathrm{Li}$ orbital. However, the left-right type of correlation required to assure proper asymptotic dependence is also present. As $\mathrm{R}$ increases this type dominates and accounts for the rapid rise in the $\sigma$ correlation energy.

TABLE 3. Square of the SOC expansion coefficient for a. LiH as a function of the internuclear distance and $b . \mathrm{BeH}^{+}$at $R=2.48$ a.u.

(a) LiH Wave Function

\begin{tabular}{|c|c|c|c|c|c|c|c|}
\hline \multirow{2}{*}{$\begin{array}{l}\text { Configu- } \\
\text { ration }\end{array}$} & \multicolumn{7}{|c|}{$\mathrm{C}^{2}(R)$} \\
\hline & $R=2.0$ & 2.6 & 3.0 & 3.4 & 4.0 & 6.0 & 8.0 \\
\hline $\begin{array}{l}2 \sigma^{2} \\
3 \sigma^{2} \\
4 \sigma^{2} \\
5 \sigma^{2}\end{array}$ & $\begin{array}{r}0.97166 \\
.01398 \\
.00208 \\
.00016\end{array}$ & $\begin{array}{r}0.97175 \\
.01396 \\
.00303 \\
.00017\end{array}$ & $\begin{array}{r}0.96989 \\
.01609 \\
.00329 \\
.00018\end{array}$ & $\begin{array}{r}0.96640 \\
.02032 \\
.00309 \\
.00019\end{array}$ & $\begin{array}{r}0.95723 \\
.03119 \\
.00238 \\
.00020\end{array}$ & $\begin{array}{r}0.85232 \\
.14324 \\
.00052 \\
.00021\end{array}$ & $\begin{array}{r}0.64815 \\
.35131 \\
.00004 \\
.00003\end{array}$ \\
\hline $\begin{array}{l}1 \pi^{2} \\
2 \pi^{2}\end{array}$ & $\begin{array}{l}.01176 \\
.00025\end{array}$ & $\begin{array}{l}.01070 \\
.00026\end{array}$ & $\begin{array}{l}.01014 \\
.00026\end{array}$ & $\begin{array}{l}.00959 \\
.00026\end{array}$ & $\begin{array}{l}.00861 \\
.00024\end{array}$ & $\begin{array}{l}.00355 \\
.00007\end{array}$ & $\begin{array}{l}.00045 \\
.00000\end{array}$ \\
\hline
\end{tabular}

(b) $\mathrm{BeH}^{+}$Wave Function

\begin{tabular}{l|r|r|r}
\hline \multicolumn{2}{c}{$R=2.48$} \\
\hline $2 \sigma^{2}$ & & 0.97320 & \\
$3 \sigma^{2}$ & .01428 & .00263 & \\
$4 \sigma^{2}$ & .00017 & .00930 & \\
$5 \sigma^{2}$ & & .00031 & \\
$1 \pi^{2}$ & & & \\
$2 \pi^{2}$ & & \\
\hline
\end{tabular}

The $p \sigma$ contribution is significant in the $4 \sigma \mathrm{PNO}$ but without plots of the orbitals it becomes increasingly difficult to ascertain the dominant correlating effects.
This orbital tends to shift charge along the bond toward the $\mathrm{Li}$.

The only other correlating orbital with a simple effect is the $1 \pi \mathrm{PNO}$, which dominates the angular correlation. As $R$ increases and charge transfers back toward the $\mathrm{Li}$, the angular correlation also becomes more diffuse and eventually goes to zero. The dependence of the $\sigma$ and $\pi$ type correlation as a function of $R$ is shown in table 4 along with the total and correlation energy.

The $\sigma$ and $\pi$ type correlation are determined by considering the $\sigma$ and $\pi$ SOC independently. As a result these estimates do not add up to the total correlation which is the difference between the total energy, for the combined $\sigma$ and $\pi$ SOC, and the H. F. energy. Such estimates are always found to exceed the true value as do the $\mathrm{C}^{2}$ coefficients. Comparison with Bender and Davidson's breakdown of the correlation is encouraging. The $\sigma$ and $\pi$ results are essentially identical. As Bender and Davidson note, this correlation breakdown does not correspond to that expected for $\mathrm{H}^{-}$.

The dissociation energy relative to the Hartree-Fock products is $2.36 \mathrm{eV}$. This is within $0.15 \mathrm{eV}$ of the experimental value of $2.51 \mathrm{eV}$ [10]. The neglect of intershell and $\delta$-type correlations cannot account for such an error. The error is also not in the convergence properties of the NO expansion for this basis. The results quoted in table 4 are for the entire basis. The PNO listed in table 2 subsume all but about $0.05 \mathrm{eV}$ of the energy contributions from this basis. The basis set is poor and will require further experimentation if an improvement is to be had.

TABLE 4. Hartree-Fock, total, and correlation energies for $\mathrm{LiH}$ and $\mathrm{BeH}^{+}$

l a.u. $($ energy $)=27.20976 \mathrm{eV}$.

\begin{tabular}{|c|c|c|c|c|c|}
\hline \multicolumn{6}{|c|}{ L.iH } \\
\hline$R$ & $-\mathrm{E}(\mathrm{H} . \mathrm{F})$. & $-\mathrm{E}($ tot.) & $-\mathrm{E} \sigma$ & $-\mathrm{E} \pi$ & $-\mathrm{E}$ (corr.) \\
\hline $\begin{array}{l}2.0 \\
2.6 \\
3.0 \\
3.4 \\
4.0 \\
6.0 \\
8.0 \\
x\end{array}$ & \begin{tabular}{|r|}
7.911640 \\
7.977167 \\
7.985388 \\
7.981539 \\
7.966088 \\
7.904227 \\
7.859542 \\
$\mathrm{a} 7.932088$
\end{tabular} & $\begin{array}{l}7.946792 \\
8.010911 \\
8.018806 \\
8.015129 \\
8.000858 \\
7.951813 \\
7.934728\end{array}$ & $\begin{array}{r}0.02125 \\
.02138 \\
.02213 \\
.02333 \\
.02598 \\
.04407 \\
.07470\end{array}$ & $\begin{array}{r}0.01668 \\
.01507 \\
.01408 \\
.01324 \\
.01220 \\
.01004 \\
.00925\end{array}$ & $\begin{array}{r}0.03515 \\
.03374 \\
.03342 \\
.03359 \\
.03473 \\
.04759 \\
.07518\end{array}$ \\
\hline \multicolumn{6}{|c|}{$\mathrm{BeH}^{+}$} \\
\hline 2.48 & 14.85108 & 14.88497 & 0.02357 & 0.01510 & 0.03489 \\
\hline
\end{tabular}

This result is the approximate Hartree-Fock energies of the atoms for the Gaussian basis used: the accurate sum of H. F. atom energies is -7.9327257 a.u. (See $\mathrm{E}$. Clementi, Tables of Atomic Functions, Supplement to IBM Journal of Research and Development 9 , 2 (1965).)

$\mathrm{BeH}^{+}$at the equilibrium configuration presents results comparable to that for equilibrium $\mathrm{LiH}$. The correlation breakdown in terms of $\sigma$ and $\pi$ contributions are essentially the same and nearly independent of the bonding characteristics of the respective Hartree-Fock molecules. The dissociation energy is $2.93 \mathrm{eV}$ and on the basis of the $\mathrm{LiH}$ calculation should be good to about $0.2 \mathrm{eV}$. 


\section{References}

[1] T. P. Das and A. C. Wahl, J. Chem. Phys. 44, 87 (1966).

[2] There is unpublished work by Das and $\mathrm{Wahl}$ on $\mathrm{F}_{2}$ and see C. Edmiston and M. Krauss, J. Chem. Phys. 45, 1833 (1966) for a PNO treatment of $\mathrm{He}_{2}^{+}$.

[3] (a) D. D. Ebbing, J. Chem. Phys. 36, 1361 (1962).

(b) S. L. Kahalas and R. K. Nesbet, J. Chem. Phys. 39, 529 (1963).

(c) F. E. Harris and H. S. Taylor, Physica 30, 105 (1964).

(d) J. C. Browne and F. A. Matsen, Phys. Rev. 135, A1227 (1964).

(e) D. D. Ebbing and R. C. Henderson, J. Chem. Phys. 42, 2225 (1965).

(f) C. F. Bender and E. R. Davidson, J. Phys. Chem. 70, 2675 (1966). (g) I. G. Czismadia, J. Chem. Phys. 44, 1849 (1966).

(h) P. E. Cade and W. M. Huo, J. Chem. Phys. 47, 614 (1967).

[4] P. O. Lowdin, Phys. Rev. 97, 1474 (1955).

[5] S. Huzinaga, J. Chem. Phys. 42, 1293 (1965).

[6] See ref [2] and R. Ahlrichs, W. Kutzelnigg, and W. A. Bingel, Teoret. Chim. Acta (Berl.) 5, 289 (1966); A. W. Weiss, Phys. Rev. to be published.

[7] P. Politzer and R. E. Brown, J. Chem. Phys. 45, 451 (1966).

[8] See [3e] and R. F. W. Bader and W. H. Henneker, J. Am. Chem. Soc. 88, 280 (1966).

[9] G. V. Nazaroff and J. O. Hirschfelder, J. Chem. Phys. 39, 715 (1963).

[10] R. Valasco, Can. J. Phys. 35, 1024 (1957).

(Paper 72Al-484) 